\title{
Acute Increases in Protein 0-GlcNAcylation Dampen Epileptiform Activity in Hippocampus
}

\author{
(ㄴ)Luke T. Stewart, ${ }^{1}$ Anas U. Khan, ${ }^{1 \star}$ Kai Wang, ${ }^{1 \star}$ Diana Pizarro, ${ }^{2}$ Sandipan Pati, ${ }^{2}$ S Susan C. Buckingham, ${ }^{3}$ \\ Michelle L. Olsen, ${ }^{1}$ John C. Chatham, ${ }^{4}$ and Lori L. McMahon ${ }^{1}$ \\ ${ }^{1}$ Department of Cell, Developmental, and Integrative Biology, ${ }^{2}$ Department of Neurology, ${ }^{3}$ Department of Neurobiology, and ${ }^{4}$ Department of Pathology, \\ University of Alabama at Birmingham, Birmingham, Alabama 35294
}

0 -GlcNAcylation is a ubiquitous and dynamic post-translational modification involving the 0 -linkage of $\beta$ - $N$-acetylglucosamine to serine/threonine residues of membrane, cytosolic, and nuclear proteins. This modification is similar to phosphorylation and regarded as a key regulator of cell survival and homeostasis. Previous studies have shown that phosphorylation of serine residues on synaptic proteins is a major regulator of synaptic strength and long-term plasticity, suggesting that 0-GlcNAcylation of synaptic proteins is likely as important as phosphorylation; however, few studies have investigated its role in synaptic efficacy. We recently demonstrated that acutely increasing 0-GlcNAcylation induces a novel form of LTD at CA3-CA1 synapses, 0-GlcNAc LTD. Here, using hippocampal slices from young adult male rats and mice, we report that epileptiform activity at CA3-CA1 synapses, generated by $\mathrm{GABA}_{\mathrm{A}} \mathrm{R}$ inhibition, is significantly attenuated when protein 0-GlcNAcylation is pharmacologically increased. This dampening effect is lost in slices from GluA2 KO mice, indicating a requirement of GluA2-containing AMPARs, similar to expression of O-GlcNAc LTD. Furthermore, we find that increasing $0-$ GlcNAcylation decreases spontaneous CA3 pyramidal cell activity under basal and hyperexcitable conditions. This dampening effect was also observed on cortical hyperexcitability during in vivo EEG recordings in awake mice where the effects of the proconvulsant pentylenetetrazole are attenuated by acutely increasing 0-GlcNAcylation. Collectively, these data demonstrate that the post-translational modification, 0-GlcNAcylation, is a novel mechanism by which neuronal and synaptic excitability can be regulated, and suggest the possibility that increasing 0-GlcNAcylation could be a novel therapeutic target to treat seizure disorders and epilepsy.

Key words: glutamate transmission; neuronal excitation; 0-GlcNAc; post-translational modification; synaptic circuits

\section{Significance Statement}

We recently reported that an acute pharmacological increase in protein 0-GlcNAcylation induces a novel form of long-term synaptic depression at hippocampal CA3-CA1 synapses (0-GlcNAc LTD). This synaptic dampening effect on glutamatergic networks suggests that increasing 0-GlcNAcylation will depress pathological hyperexcitability. Using in vitro and in vivo models of epileptiform activity, we show that acutely increasing 0-GlcNAc levels can significantly attenuate ongoing epileptiform activity and prophylactically dampen subsequent seizure activity. Together, our findings support the conclusion that protein 0-GlcNAcylation is a regulator of neuronal excitability, and it represents a promising target for further research on seizure disorder therapeutics.

\section{Introduction}

Protein phosphorylation is a primary regulator of neuronal function, and an extensive literature exists establishing critical roles of

Received Jan. 15, 2016; revised July 2, 2017; accepted July 8, 2017.

Author contributions: L.T.S., K.W., S.C.B., M.L.O., J.C.C., and L.L.M. designed research; L.T.S., A.U.K., K.W., and S.C.B. performed research; M.L.O. contributed unpublished reagents/analytic tools; L.T.S., A.U.K., K.W., S.P., D.P., S.C.B., J.C.C., and L.L.M. analyzed data; L.T.S., K.W., S.P., J.C.C., and L.L.M. wrote the paper.

This work was supported by National Institutes of Health National Institute of Neurological Disorders and Stroke R01NS076312 to L.L.M. and J.C.C. and 1F31NS095568 to L.T.S.

The authors declare no competing financial interests.

*A.U.K. and K.W. contributed equally to this work.

Correspondence should be addressed to Dr. Lori L. McMahon, University of Alabama at Birmingham, Department of Cell, Developmental and Integrative Biology, 1918 University Blvd, MCLM 988, Birmingham, AL 35294. E-mail: mcmahon@uab.edu. serine phosphorylation/dephosphorylation in regulating longterm synaptic plasticity and learning and memory (Anggono and Huganir, 2012; Lüscher and Malenka, 2012). Whereas phosphorylation is a well-established and extensively characterized posttranslational modification, O-GlcNAcylation, the O-linkage of the monosaccharide $\beta$ - $N$-acetylglucosamine to serine/threonine residues, is increasingly recognized as a major regulator of pro-

S.C. Buckingham's present address: Birmingham-Southern College, 900 Arkadelphia Road, Birmingham, AL 35254.

M.L. Olsen's present address: Virginia Polytechnic Institute and State University, School of Neuroscience, 970 Washington Street SW, Blacksburgh, VA 24061.

DOI:10.1523/JNEUROSCI.0173-16.2017

Copyright $\odot 2017$ the authors $\quad 0270-6474 / 17 / 378207-09 \$ 15.00 / 0$ 
tein function (Bond and Hanover, 2015). Unlike the numerous kinases and phosphatases regulating phosphorylation, the O-GlcNAc post-translational modification is regulated by two enzymes: O-GlcNAc transferase (OGT), which adds the modification; and O-GlcNAcase (OGA), which catalyzes its removal. The donor metabolite for O-GlcNAcylation is the molecule UDP-GlcNAc, which is the second most abundant high-energy metabolite available next to ATP (Hart, 2014). Synthesis of UDP-GlcNAc by the hexosamine biosynthetic pathway (HBP) incorporates ATP, glucose, acetyl-CoA, and uridine, supporting the notion that protein O-GlcNAcylation serves as a molecular sensor capable of detecting fluctuations in global metabolism on a rapid timescale (Bond and Hanover, 2015). Thus, the HBP is a prime pathway to meet the energy needs of active neural tissue, whose complex activity has an enormous metabolic demand.

The brain exhibits the second highest abundance of O-GlcNAcylated proteins in the body, behind the pancreas (Parker et al., 2011; Trinidad et al., 2012), with markedly dense expression in hippocampus and cerebellum (Liu et al., 2004). A host of O-GlcNAcylated proteins are contained in both the presynaptic and postsynaptic compartments (Parker et al., 2011; Trinidad et al., 2012). Despite these findings, the majority of studies in brain have focused on O-GlcNAcylation in the context of Alzheimer's disease and other neuropathies (Schulz, 2008; Lagerlöf and Hart, 2014; Wang et al., 2016; Yang et al., 2017; Yuzwa and Vocadlo, 2014; Yuzwa et al., 2014), with relatively few studies investigating the mechanisms by which O-GlcNAcylation modulates neuronal and circuit function.

Recently, we reported a novel form of long-term synaptic depression at hippocampal CA3-CA1 glutamatergic synapses driven by acute increases in protein O-GlcNAcylation, which we termed O-GlcNAc LTD (Taylor et al., 2014). Mechanistically, our findings show a requirement for the AMPAR GluA2 subunit during expression of O-GlcNAc LTD. In addition to inducing synaptic LTD, increasing O-GlcNAcylation disrupts LTP expression at CA3-CA1 synapses. Furthermore, rats injected with the highly selective OGA inhibitor Thiamet G (TMG) (Macauley et al., 2005; Yuzwa et al., 2008) to elevate brain O-GlcNAc levels in vivo exhibited deficits in both the novel object placement (NOP) and novel object recognition (NOR) tasks, but not in contextual fear conditioning, compared with saline controls (Taylor et al., 2014). In addition to the AMPAR GluA2 subunit, other publications have identified additional neuronal proteins, which are O-GlcNAcylated, including Synapsin I/II (Tallent et al., 2009), CREB (Rexach et al., 2012), and KCNQ3 (Ruan et al., 2014), all exerting a modulatory effect on neural function. Recently, the $\mathrm{OGA}^{+/-}$transgenic mouse was reported to have impaired learning and memory together with reduced LTP and LTD (Yang et al., 2017). Chronic elevations in O-GlcNAcylation were shown in these mice as well as disruptions in chemically induced AMPAR GluA1 phosphorylation, presumably a result of dysregulated crosstalk between O-GlcNAc and phosphorylation.

Based upon our previous finding that acute increases in O-GlcNAcylation induce LTD, we hypothesize that increased O-GlcNAcylation might be capable of dampening pathological hyperexcitability, such as occurs during seizure activity and epilepsy. Here, we demonstrate that acutely increasing O-GlcNAcylation via application of the HBP substrate glucosamine, either alone or in combination with the OGA inhibitor TMG, significantly reduces epileptiform activity in vitro in both adult rat and mouse hippocampal slices, as well as in vivo during acute seizure activity in awake behaving mice. Collectively, these data demonstrate that increasing O-GlcNAcylation is capable of dampening acute epileptiform activity. Thus, increasing O-GlcNAcylation may be a viable therapeutic strategy to limit hyperexcitability underlying pathological conditions, such as epilepsy and other seizure disorders.

\section{Materials and Methods}

All experimental procedures were approved by the University of Alabama at Birmingham Institutional Animal Care and Use Committee and follow the National Institute of Health experimental guidelines.

Hippocampal slice preparation. Young adult male Sprague Dawley rats (age 6-10 weeks; Charles River Laboratories) and adult male C57BL/6 mice (6-8 weeks old; Charles River Laboratories) were anesthetized with isoflurane, decapitated, and brains removed; $400 \mu \mathrm{m}$ coronal slices from dorsal hippocampus were made on a VT1000P vibratome (Leica Biosystems) in oxygenated $\left(95 \% \mathrm{O}_{2} / 5 \% \mathrm{CO}_{2}\right)$ ice-cold high sucrose cutting solution (in mM as follows: $85.0 \mathrm{NaCl}, 2.5 \mathrm{KCl}, 4.0 \mathrm{MgSO}_{4}, 0.5 \mathrm{CaCl}_{2}, 1.25$ $\mathrm{NaH}_{2} \mathrm{PO}_{4}, 25.0$ glucose, 75.0 sucrose). After cutting, slices were held at room temperature from 1 to $5 \mathrm{~h}$ in a submersion chamber with continuously oxygenated standard ACSF (in mM as follows: $119.0 \mathrm{NaCl}, 2.5$ $\mathrm{KCl}, 1.3 \mathrm{MgSO}_{4}, 2.5 \mathrm{CaCl}_{2}, 1.0 \mathrm{NaH}_{2} \mathrm{PO}_{4}, 26.0 \mathrm{NaHCO}_{3}, 11.0$ glucose).

AMPAR GluA2 mutant mice. C57BL/6J Griat ${ }^{\text {tmlRod }}$ mice (RRID: 2176810) were purchased from The Jackson Laboratory. Heterozygous mating pairs were established, and both male and female WT and KO were used for electrophysiological recordings at 1-2 months of age. PCR was used for genotyping using the primer sequences: WT $5^{\prime}$-GGTTGGT CACTCACCTGCTT-3'; KO 5' -GCCTGAAGAACGAGATCAGC-3'

Electrophysiology. All recordings were performed in a submersion chamber with continuous perfusion of standard ACSF. Extracellular CA1 pyramidal population spikes (PSs; $0.6-1.2 \mathrm{mV}$ baseline amplitude) were recorded using a glass pipette placed in stratum pyramidale and evoked using a twisted nichrome wire (A\&M Systems) stimulating electrode positioned in CA1 stratum radiatum to activate Schaffer collateral axons $(0.1 \mathrm{~Hz}, 100 \mu$ s duration). In vitro epileptiform activity was induced using bath application of the $\mathrm{GABA}_{\mathrm{A}} \mathrm{R}$ antagonist picrotoxin (PTX, 50-100 $\mu \mathrm{M}$; Sigma). Evoked PSs were acquired using pClamp 10.2 (Molecular Devices), and CA1 hyperexcitability was quantified using a modified version of the Coastline Burst Index (mCBI) (Korn et al., 1987). The equation, $m C B I=\sum_{25}^{125}\left(\left|V_{x+1}-V_{X}\right|\right)$, summates the voltage differential between each point in the waveform (sampled at $10 \mathrm{k} \mathrm{Hz}$ ) beginning $5 \mathrm{~ms}$ after the stimulus artifact $(25 \mathrm{~ms})$ and encompassing all repetitive PS activity (25-125 ms corresponding to 2000 total data points per waveform). Values were normalized to baseline recording in control conditions. Extracellular CA3 spontaneous PS activity was recorded using a glass pipette positioned in CA3 stratum pyramidale. CA3 PS events were detected and quantified using Clampex 10.2 (Molecular Devices) automated waveform template identification functions built on the first 100 hand-selected spikes for each recording.

Modulation of O-GlcNAc levels. Acute increase in O-GlcNAcylation in vitro was induced via bath application of glucosamine $(\mathrm{GlcN}, 5 \mathrm{~mm}$; Sigma) alone or in combination with the selective OGA inhibitor TMG ( $1 \mu \mathrm{M}$; Chem Molecules), the most potent OGA inhibitor $\left(K_{\mathrm{i}}=21 \mathrm{nM}\right.$; human OGA) currently available (Macauley et al., 2005; Yuzwa et al., 2008). In vivo increase in global O-GlcNAcylation was achieved using intraperitoneal injections of both GlcN (300 mg/kg) and TMG $(10 \mathrm{mg} / \mathrm{kg})$. In both in vitro and in vivo experiments, GlcN and TMG were used in combination to ensure robust and lasting increases in protein O-GlcNAc levels during prolonged hyperexcitable conditions, which itself can alter neuronal glucose utilization (Ivanov et al., 2015) and possibly affect HBP synthesis of UDP-GlcNAc. In some experiments, we tested whether applying the HBP substrate GlcN alone was sufficient to modulate PTXinduced hyperexcitability.

Western blot analysis. Western blot analysis of protein O-GlcNAcylation in isolated CA1 regions was performed using the CTD 110 O-GlcNAc antibody as previously described (Comer et al., 2001; Zou et al., 2012; Taylor et al., 2014). Briefly, protein concentrations of CA1 homogenates were determined using colorimetric analysis assay (Bio-Rad Laboratories). Samples were loaded at $35 \mu \mathrm{g}$, separated on $7.5 \%$ SDS-PAGE, and transferred overnight to PVDF immunoblot membranes. Immunoblots were incubated with specified primary antibodies at $4^{\circ} \mathrm{C}$ overnight. After 
3 TBST/PBS washes, immunoblots were incubated with secondary antibodies at room temperature for $1 \mathrm{~h}$, followed by 3 TBST/PBS washes. Immunoblots were visualized using enhanced chemiluminescence (PerkinElmer).

EEG recordings and in vivo seizure activity. Epidural electrode implantation surgery was performed as previously described (Robel et al., 2015). Briefly, adult C57BL/6 mice were each implanted with one singlechannel electrode (Plastics ONE) lowered through unilaterally drilled holes into the rostral parietal plate. Duralay cement (Reliance Dental) and three 0.060 inch machine-threaded anchor screws were used to secure the electrode. After $5 \mathrm{~d}$ of postoperative recovery, basal cortical activity was recorded for $24 \mathrm{~h}$. The next morning, mice were injected with GlcN+TMG $(300 \mathrm{mg} / \mathrm{kg}+10 \mathrm{mg} / \mathrm{kg}$, i.p. $)$ or saline. Three hours later, all mice received the $\mathrm{GABA}_{\mathrm{A}} \mathrm{R}$ antagonist pentylenetetrazole $(\mathrm{PTZ}, 40 \mathrm{mg} / \mathrm{kg}$; Sigma) to induce hyperexcitability. EEG data were acquired using Biopac Systems amplifiers (Biopac Systems EEG100C) sampling at $500 \mathrm{~Hz}$ and AcqKnowledge 4.2 EEG Acquisition and Reader Software (Biopac Systems).

Quantification of interictal spikes and seizure activity. Determination of seizure dynamics and quantification of interictal spike activity were performed using automated line-length analysis (Esteller et al., 2005). Interictal spikes were defined as transient high amplitude deflections lasting 20-70 ms. Threshold for seizure onset was set manually during pilot analysis, then applied to all seizure events using a $2 \mathrm{~s}$ sliding window. To avoid bias during spike selection caused by electrical noise and behaviorally associated spike artifacts (e.g., grooming), an automated spike detection method was implemented. In this modified line-length analysis, higher-order derivatives are used to amplify voltage differentials in the $2 \mathrm{~s}$ sliding window. For this study, a detection threshold of 10 SDs above baseline was used to quantify interictal spikes. Spectral analysis was performed using fast Fourier transform algorithms in MATLAB (The MathWorks). Power spectrum densities were calculated from $10 \mathrm{~s}$ epochs across different seizure stages, with spectra divided into individual frequency bands corresponding to delta $(0.5-4 \mathrm{~Hz})$, theta $(4-8 \mathrm{~Hz}), \alpha(8-12 \mathrm{~Hz})$, $\beta(15-30 \mathrm{~Hz})$, and lower gamma $(24-80 \mathrm{~Hz})$.

Experimental design and statistical analysis. Slice electrophysiology data were compared using unpaired $t$ test as indicated. All nontransgenic rats and mice were male only, with slices recorded from a minimum of 5 animals from each group. Experiments involving GluA2 mutant mice included both male and female mice, with no sex difference detected. Spontaneous population activity in area CA3 was analyzed using the Kolmogorov-Smirnov (KS) test. Western blot data were compared using one-way ANOVA with Tukey's post hoc test. EEG data were compared using two-way ANOVA or paired $t$ test as indicated. All data were analyzed using GraphPad Prism 7 and Excel 2016. Data are presented as mean \pm SEM, and statistical differences were considered significant where $p<0.05$.

\section{Results}

Increasing O-GlcNAcylation in vitro dampens ongoing epileptiform activity in area CA1

To characterize the effects of increasing protein O-GlcNAcylation on sustained epileptiform activity, hippocampal slices were exposed to the $\mathrm{GABA}_{\mathrm{A}} \mathrm{R}$ antagonist PTX to induce a stable and ongoing hyperexcitable state (Fig. $1 A$, solid bar, inset, baseline in black, PTX in red). During the application of PTX, PS morphology changed from a single synchronous CA1 discharge, a product of potent feedforward GABAergic inhibition, into higher-amplitude asynchronous poly-spikes where PTX-mediated $\mathrm{GABA}_{\mathrm{A}} \mathrm{R}$ blockade allowed for repeated and dysregulated firing of the CA1 pyramidal cell population. To test the efficacy of acutely increasing O-GlcNAcylation in dampening this hyperexcitability, GlcN+TMG was coapplied $20 \mathrm{~min}$ after the start of PTX (Fig. $1 A_{1}$, bottom dashed bar) and was maintained for the duration of the experiment, in continued presence of PTX. This intervention caused a significant reduction in PTX induced CA1 hyperexcitability, measured as a decrease in $\mathrm{mCBI}$, in hippocampal slices from both rat (Fig. $1 A_{1}$; normalized mCBI of $2.2 \pm 0.2$ in PTX alone vs $1.8 \pm$
0.2 in PTX + GlcN + TMG, $p<0.05$ ) and mouse (Fig. $1 B_{1}$; normalized mCBI of $1.9 \pm 0.2$ in PTX alone vs $1.6 \pm 0.1$ in PTX+ GlcN+TMG, $p<0.05)$.

To determine whether prolonged hyperexcitability itself alters basal O-GlcNAcylation, and to determine whether GlcN+TMG significantly increases $\mathrm{O}-\mathrm{GlcN}$ Acylation during hyperexcitable conditions, we performed Western blot analysis of isolated CA1 regions from rat hippocampal slices subjected to the same pharmacological protocols as in Figure $1 A_{1}$ but in the absence of electrical stimulation. We found no difference in global O-GlcNAcylation between control and PTX (Fig. $1 C$; relative O-GlcNAc levels in vehicle of $1 \pm 0.0$ vs $1.08 \pm 0.08$ in PTX, $n=8, p>0.05)$. However, GlcN + TMG was able to significantly increase in O-GlcNAcylation during a prolonged hyperexcitable state in PTX (Fig. $1 C$; relative O-GlcNAc levels in PTX of $1.08 \pm 0.08$ vs $1.55 \pm 0.12$ in PTX + GlcN + TMG, $n=8, p<0.05)$.

\section{Pretreatment with GlcN + TMG dampens subsequently induced epileptiform activity in area CA1}

To determine whether increasing O-GlcNAcylation before PTX exposure could attenuate subsequent hyperexcitability, slices were preincubated in GlcN+TMG for $30 \mathrm{~min}$ before beginning recordings. A stable baseline of evoked PS amplitude was established followed by bath application of PTX to induce prolonged hyperexcitability. GlcN+TMG-pretreated slices showed significantly attenuated $\mathrm{mCBI}$, reflecting decreased hyperexcitability upon PTX exposure compared with nonpretreated slices. This dampening effect on hyperexcitability was observed in both rat (Fig. $1 A_{2}$; normalized mCBI of $1.8 \pm 0.1$ in GlcN+TMGpretreated + PTX vs $2.2 \pm 0.2$ in PTX alone, $p<0.05)$ and mouse (Fig. $1 B_{2}$; normalized $\mathrm{mCBI}$ of $1.3 \pm 0.1$ in GlcN+TMGpretreated + PTX vs $1.9 \pm 0.1$ in PTX alone, $p<0.05$ ) hippocampal slices.

To test the sustainability of elevated O-GlcNAcylation during prolonged hypexcitability, slices first underwent a $30 \mathrm{~min}$ preincubation in GlcN+TMG, then a subsequent 40 min exposure to PTX alone. We found that $\mathrm{O}-\mathrm{GlcNA}$ cylation was significantly elevated in the GlcN+TMG-pretreated slices following prolonged PTX exposure compared with PTX alone (Fig. $1 D$; relative O-GlcNAc levels in PTX of $0.88 \pm 0.06$ vs $1.27 \pm 0.10$ in PTX + GlcN + TMG, $n=12$, $p<0.05)$.

\section{Glucosamine alone dampens epileptiform activity in CA1}

Given the positive effects of combined GlcN+TMG application on hyperexcitability, we next wanted to determine whether GlcN alone could dampen hyperexcitability because of the possibility of easy translation to humans since $\mathrm{GlcN}$ is an over-the-counter dietary supplement. Similar to the experiment in Figure $1 A_{l}$, hyperexcitability was induced in slices with bath application of PTX followed by application of GlcN alone that continued for the duration of the experiment. Importantly, GlcN induced a significant reduction in hyperexcitability measured as a decrease in $\mathrm{mCBI}$ (Fig. 2A; normalized mCBI of $2.0 \pm 0.2$ in PTX alone vs $1.6 \pm 0.1$ in $\mathrm{PTX}+\mathrm{GlcN}, p<0.05)$.

\section{Brief glucosamine exposure is sufficient to dampen subsequent epileptiform activity}

We previously reported that a $10 \mathrm{~min}$ bath application of GlcN induces a novel form of LTD (O-GlcNAc LTD) at CA3-CA1 synapses. The long-term expression of O-GlcNAc LTD (>60 min after induction) outlasts the transient increase in protein O-GlcNAcylation that occurs with a 10 min GlcN exposure because O-GlcNAc levels return to baseline within $10-20 \mathrm{~min}$ following $\mathrm{GlcN}$ washout. 

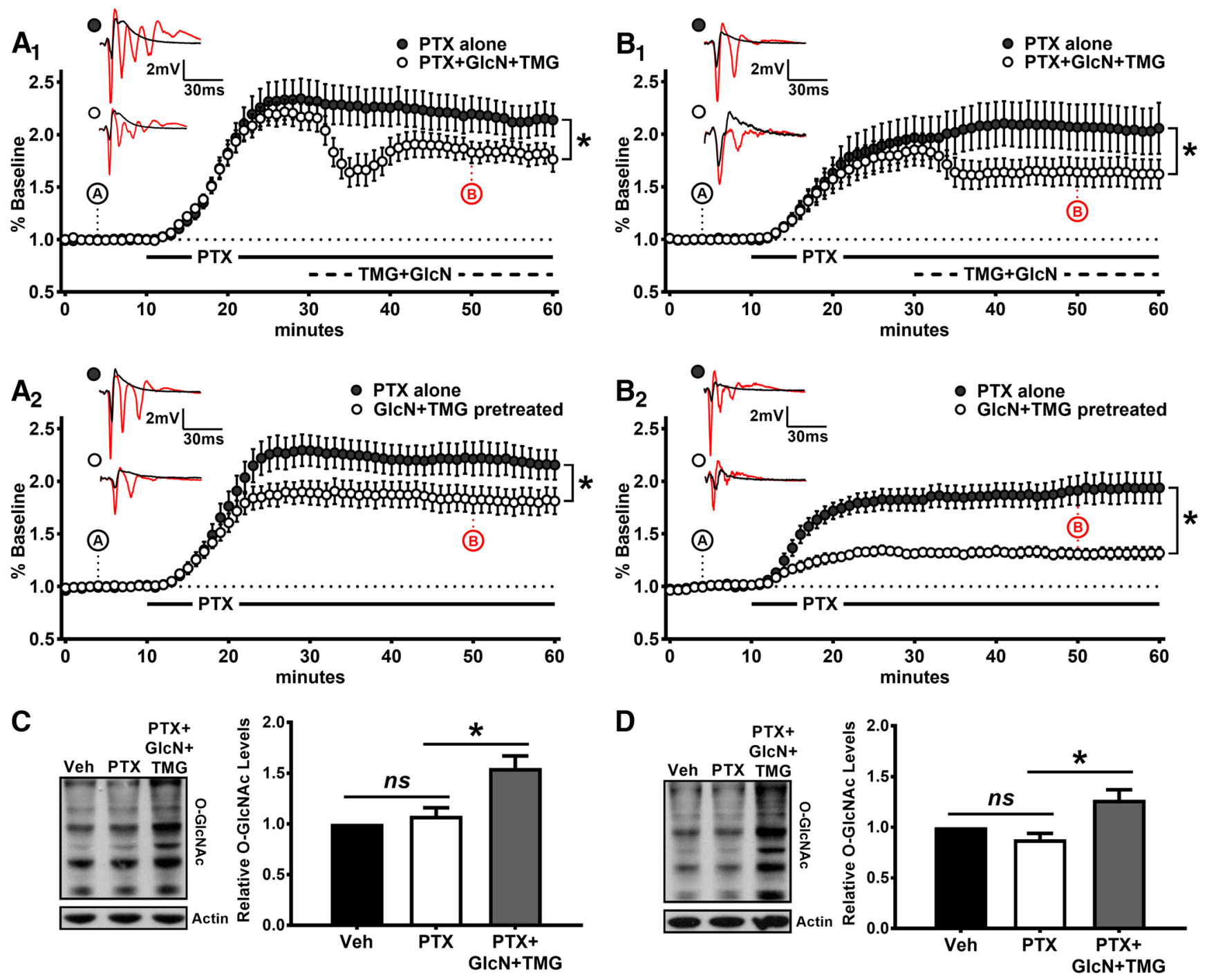

Figure 1. Pharmacologically increased 0 -GlcNAcylation attenuates in vitro epileptiform activity in hippocampus. $A_{1}$, Summary plot of rat hippocampal slices exposed to PTX (min $10-60$ ) followed by bath application of GICN + TMG (min 30-60) showing significant reduction in CA1 hyperexcitability (PTX+GIcN + TMG, $n=12$ slices vs PTX alone, $n=9$ slices, $p<0.05$ ). $A_{2}$, Summary plot of rat hippocampal slices pretreated with GICN + TMG for 30 min followed by bath application of PTX ( $\left.\min 10-60\right)(G I c N+$ TMG pretreated + PTX, $n=7$ slices vs PTX alone, $n=$ 14 slices, $p<0.05$ ). $\boldsymbol{B}_{1}$, Summary plot of mouse hippocampal slices exposed to PTX ( $\left.\min 10-60\right)$ followed by bath application of GIcN + TMG (min $30-60$ ) showing a significant reduction in CA1 hyperexcitability (PTX + GIcN + TMG, $n=14$ slices vs PTX alone, $n=11$ slices $p<0.05$ ). $B_{2}$, Summary plot of mouse hippocampal slices pretreated with GlcN + TMG for 30 min followed by bath application ( $\min 10-60$ ) of PTX (GICN + TMG pretreated + PTX, $n=7$ slices vs PTX alone, $n=9$ slices, $p<0.05$ ). C, Western blot analysis of rat hippocampal slices exposed to 40 min of PTX with the addition of GICN + TMG 20 min into PTX exposure to recapitulate the pharmacological protocol in $\boldsymbol{A}$ (PTX + GICN + TMG vs PTX alone, $n=8, p<0.05$ ). $\boldsymbol{D}$, Western blot analysis of rat hippocampal slices treated for $30 \mathrm{~min}$ in GIcN + TMG followed by $40 \mathrm{~min}$ of PTX exposure to recapitulate the pharmacological protocol in $\boldsymbol{B}$ (GIcN + TMG-pretreated + PTX vs PTX alone, $n=12$, $p<0.05) .{ }^{*} p<0.05$.

Thus, O-GlcNAc LTD expression does not require a continuous increase in protein O-GlcNAcylation (Taylor et al., 2014). Therefore, we next sought to determine whether the O-GlcNAc-induced dampening of hyperexcitability could similarly occur as a consequence of short-term GlcN exposure and transient increase in protein O-GlcNAcylation, or if continuous GlcN exposure, as in Figure $2 A$, is required, which will maintain increased protein O-GlcNAcylation. Following a stable baseline, a $10 \mathrm{~min}$ bath application of GlcN induced significant depression of the PS, reflecting O-GlcNAc LTD (Fig. $2 B$, inset graph; normalized mCBI of $1.01 \pm 0.009$ in PTX vs $0.89 \pm 0.02$ in GlcN-PTX, $p<0.05)$. After a $10 \mathrm{~min}$ GlcN washout, PTX was applied to induce hyperexcitability, and maximum mCBI was compared with that measured in slices not pre-exposed to GlcN. We found that the maximum mCBI was significantly reduced, reflecting decreased hyperexcitability, in slices pre-exposed to GlcN and expressing
O-GlcNAc LTD, compared with slices not previously exposed to GlcN (Fig. 2B; normalized mCBI of $1.65 \pm 0.11$ in GlcN-PTX compared with $2.14 \pm 0.29$ in PTX alone, $p<0.05$ ). These results show that a short GlcN exposure that causes a transient increase in O-GlcNAcylation and induces O-GlcNAc LTD (Taylor et al., 2014) is capable of dampening subsequently induced hyperexcitability.

O-GlcNAc-mediated dampening of epileptiform activity is attenuated in mice lacking GluA2 AMPAR subunits We previously showed a requirement for the GluA2 subunit of AMPARs for O-GlcNAc LTD at CA3-CA1 synapses (Taylor et al., 2014). To further investigate the possibility of a conserved GluA2 requirement between O-GlcNAc LTD with the dampening of hyperexcitability in area CA1, slices from mice lacking functional GluA2 AMPAR subunits (C57BL/6J Gria2 ${ }^{\text {tm1-Rod }}$ ) were exposed to PTX to induce a stable epileptiform state followed by bath appli- 

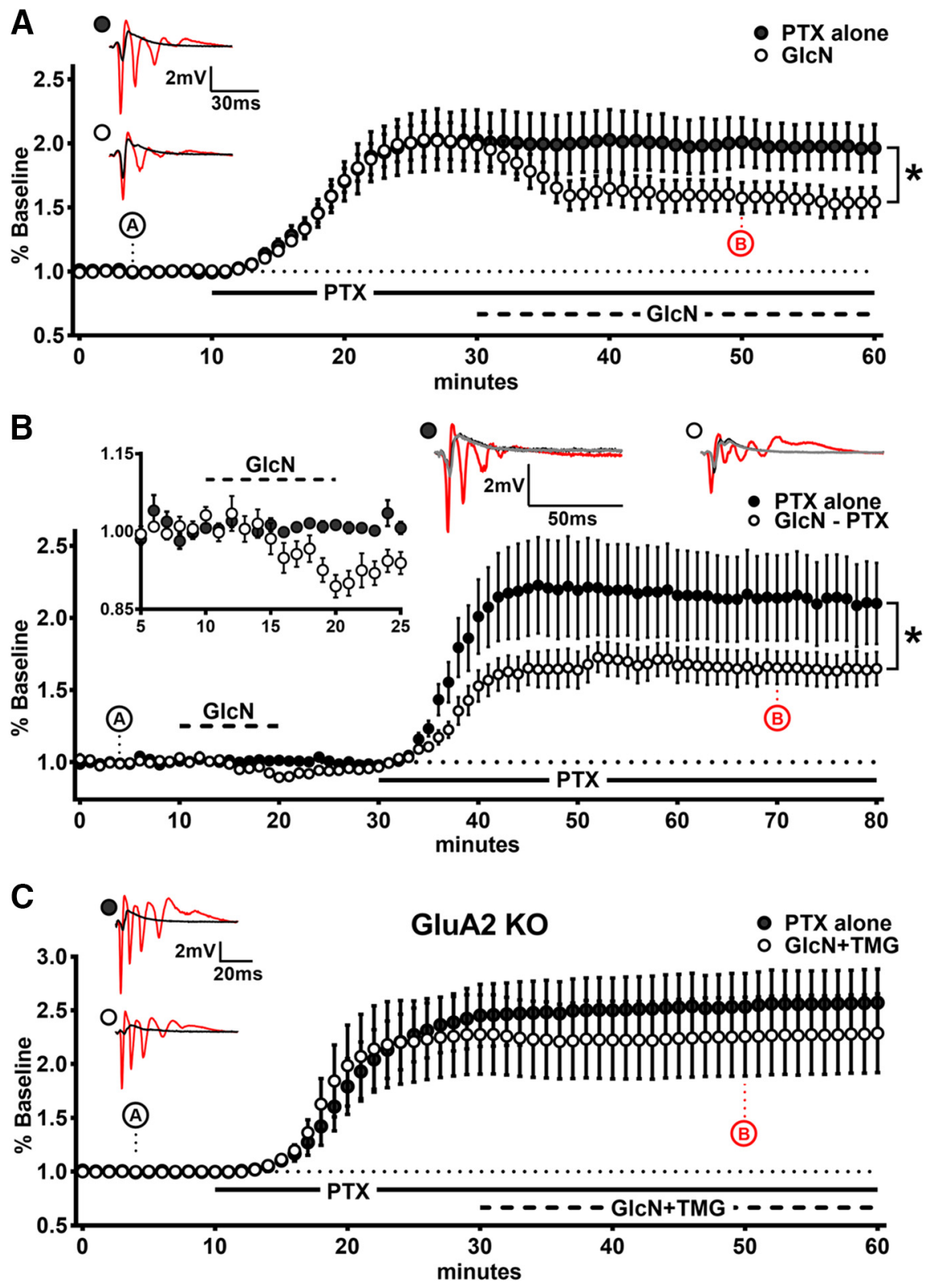

Figure 2. 0-GICNAc-mediated dampening of epileptiform activity is induced by glucosamine alone, can occur with only shortterm glucosamine exposure, and requires AMPAR GluA2 subunits. $A$, Summary plot of rat hippocampal slices exposed to PTX (min 10 - 60) followed by bath application of GlcN ( $\min 30-60$ ) showing significant reduction in CA1 hyperexcitability (PTX+GlcN, $n=6$ slices vs PTX alone, $n=5$ slices, $p<0.05$ ). All waveforms correspond to baseline (time point A; black, $5 \mathrm{~min}$ ) or late-stage pharmacological treatment (time point $B$; red, $50 \mathrm{~min}$ ). $\boldsymbol{B}$, Summary plot of rat hippocampal slices transiently depressed by $10 \mathrm{~min}$ application of GICN ( $\min 10-20)$ followed by 10 min washout before exposure to PTX ( $\min 30-80$ ) showing significant reduction in hyperexcitability (PTX alone, $n=9$ slices vs GlcN-PTX, $n=14$ slices, $p<0.05$ ). C, Summary plot showing no significant dampening of PTX-induced hyperexcitability in slices from GluA2 KO mice during GICN + TMG application ( $\min 30-60$ ) compared with PTX alone recorded in interleaved experiments (PTX alone, $n=11$ slices from 5 mice vs PTX + GlcN + TMG, $n=8$ slices from 5 mice, $p>0.05) .{ }^{*} p<0.05$.

cation of GlcN + TMG in continued PTX to increase O-GlcNAc levels. PTX-exposed slices treated with GlcN+TMG showed no significant decrease in hyperexcitability compared with PTX alone slices (Fig. $2 C$; normalized $\mathrm{mCBI}$ of $2.5 \pm 0.31$ in PTX alone vs $2.25 \pm 0.37$ in PTX + GlcN + TMG, $p=0.29$ ), indicating a requirement for GluA2-containing AMPARs for the expression of O-GlcNAc dampening of hyperexcitability. Importantly, slices from WT littermate control mice were interleaved to confirm dampening of PTX-induced hyperexcitability by GlcN+TMG (normalized mCBI of $1.96 \pm 0.21$ in PTX alone vs $1.44 \pm 0.14$ in PTX + GlcN + TMG, $p<0.05$; data not shown)

Increased O-GlcNAc levels dampen basal spontaneous $\mathrm{CA} 3$ pyramidal cell network activity as well as PTX-induced hyperexcitability

CA3 pyramidal cells are spontaneously active due to recurrent collateral innervation (Cohen and Miles, 2000). Therefore, we next asked whether acutely increasing O-GlcNAcylation dampens spontaneous network activity. As shown in the cumulative distribution plots, we found that bath application of GlcN alone reduces basal CA3 spike frequency (Fig. $3 A_{1}$; mean interevent interval of $848 \mathrm{~ms}$ during basal conditions vs $1231 \mathrm{~ms}$ in PTX+GlcN, $p<$ 0.01 ; KS-Z score: 10.098). This effect is shown in voltage traces of basal CA3 activity (Fig. $3 A_{2}$ ), which exhibit normal population spike characteristics (Fig. $3 A_{3}$ ) and significant reductions in spontaneous activity upon GlcN administration (Fig. $3 A_{4}$ ). Importantly, GlcN also decreased the PTX-induced increase in CA3 spike frequency (Fig. $3 B_{1}$; mean interevent interval in PTX, $378 \mathrm{~ms}$ vs in PTX $+\mathrm{GlcN}$, $516 \mathrm{~ms}, p<0.01$; KS-Z: 4.746). This dampening effect of increased O-GlcNAc is shown in voltage traces from PTX alone (Fig. $3 B_{2}$ ) compared with $\mathrm{PTX}+\mathrm{GlcN}$ (Fig. $3 B_{3}$ ).

Increasing O-GlcNAc reduces cortical epileptiform activity in vivo

The dampening of PTX-induced hyperexcitability observed in the brain slice electrophysiology experiments following an increase in O-GlcNAcylation prompted us to ask whether this dampening effect could also be observed in vivo. Because increasing O-GlcNAcylation dampens but does not inhibit ongoing epileptiform activity or prevent subsequently induced epileptiform activity, we predicted that it should be able to attenuate interical spike activity, and it might even alter the frequency spectra during seizure activity, but may not fully prevent seizures. To test these ideas, mice were fitted with a singlechannel epidural cortical EEG electrode and injected with saline or GlcN+TMG (300 mg/kg and $10 \mathrm{mg} / \mathrm{kg}$, i.p., respectively) to increase brain O-GlcNAc levels. Three hours after saline or GlcN+TMG injection, all animals were challenged with an intraperitoneal injection of the $\mathrm{GABA}_{\mathrm{A}} \mathrm{R}$ antagonist PTZ ( $40 \mathrm{mg} / \mathrm{kg}$ ), a model often used to screen for anticonvulsant efficacy of experimental antiepileptic drugs (Löscher, 2009). Initial analysis focused on PTZinduced increases in interictal spikes, where PTZ significantly increased interictal spikes in saline-pretreated animals (Fig. 4A; baseline control vs PTZ, $p<0.05$ ). Importantly, increasing 
O-GlcNAcylation with GlcN+TMG before the PTZ injection prevented the PTZinduced increase in interictal spikes that was observed in the saline-pretreated cohort (Fig. 4A; baseline GlcN + TMG vs PTZ GlcN+TMG, $p>0.05)$. GlcN+TMG pretreatment had no effect on latency to initial interictal spike (saline latency of $222 \pm 69 \mathrm{~s}$ vs $240 \pm 56 \mathrm{~s}$ in GlcN+TMGpretreated mice, $p>0.05$; data not shown).

Although all animals exhibited interictal spike activity, a subset of mice in each treatment group also exhibited full convulsive seizures (control, $n=7$ of 15 ; GlcN+TMG, $n=5$ of 14 ; all electrographic seizures corresponding to confirmed Racine Stage 5 behavior: supine arched head/tail with forelimb tonus/ clonus). Spectral analysis reveals altered frequency distributions of seizure events between GlcN+TMG-pretreated animals and saline controls, where seizures in GlcN+ TMG-treated animals exhibit maximum power in lower frequency domains (Fig. 4B, gray curve, top arrow) compared with seizures in saline-treated animals, which show prevalence across higher frequency domains (Fig. 4B, black curve, bottom arrow). Line-length analysis was used to calculate seizure initiation and termination times to determine seizure duration, which showed no significant difference across groups (Fig. 4C; control seizure duration of $20.37 \pm 0.4 \mathrm{~s}$ vs $19.34 \pm 3.08 \mathrm{~s}$ in GlcN+TMG-pretreated, $p>0.05)$. Additionally, latency to initial seizure showed no difference across groups (saline latency of $420 \pm 67$ vs $339 \pm$ $132 \mathrm{~s}$ in GlcN+TMG-pretreated mice, $p>0.05$; data not shown), suggesting no change in seizure threshold as a result of GlcN+TMG treatment.

Analysis of frequency dynamics during seizure progression shows altered profiles in saline versus GlcN+TMG-treated mice. In a saline-treated mouse, the seizure (Fig. $4 D_{2}$; with accompanying energy profile in $4 \mathrm{D}_{3}$ and power spectrum density [PSD] plot in Fig. $4 D_{4}$ ) shows a distinct departure from delta band predominance (Fig. $4 D_{1}$; Epoch 1, blue bar) toward higher frequency bands during ictal activity (Fig. $4 D_{1}$; Epoch 3, green and orange bars). In contrast, in a GlcN+TMG-treated mouse, the seizure (Fig. $4 E_{2}$; with accompanying energy profile in $4 \mathrm{E}_{3}$ and PSD plot in Fig. $4 E_{4}$ ) exhibits the opposite frequency redistribution where pre-ictal activity shows typical delta band predominance (Fig. $4 B_{1}$; Epoch 1) followed by left-shifted frequency distribution and even higher delta band predominance during fully ictal seizure progression (Fig. $4 B_{1}$; Epochs 3 and 4). Recent studies suggest that specific electrographic patterns of seizure activity might be useful as biomarkers for epilepsy disease progression (Dudek and Staley, 2011; Ge et al., 2013; Milikovsky et al., 2017). Therefore, interventions that alter the electrographic profile, as shown above with increased O-GlcNAcylation, could be beneficial in modifying disease progression and, as such, require extensive future investigation.

Western blot analysis revealed significant increases in O-GlcNAc levels in GlcN+TMG-treated mice compared with saline-treated control mice (Fig. $4 F_{2}$; vehicle $1.0 \pm 0.14$, PTZ, $1.02 \pm 0.01$, $\mathrm{PTZ}+\mathrm{GlcN}+\mathrm{TMG}, 1.83 \pm 0.07, p<0.05)$, but there was no significant difference in hippocampal OGT expression (Fig. $4 F_{3}$; vehicle, $1.0 \pm 0.03, \mathrm{PTZ}, 1.02 \pm 0.04, \mathrm{PTZ}+\mathrm{GlcN}+\mathrm{TMG}, 1.12 \pm$ $0.04, p>0.05)$.

\section{Discussion}

Despite the increasing number of neuronal proteins identified as being O-GlcNAcylated, there are relatively few reports examining the physiological role of O-GlcNAcylation in modulating neuronal function or synaptic transmission/plasticity (Tallent et al., 2009; Kanno et al., 2010; Ruan et al., 2014; Ogawa et al., 2015; Taylor et al., 2014; Wang et al., 2016; Yang et al., 2017). Previously, we reported that acutely increasing O-GlcNAcylation induces a novel NMDAR-independent, long-lasting depression of basal synaptic strength at CA3-CA1 hippocampal synapses (O-GlcNAc LTD) as well as a disruption of tetanus-induced LTP at these same glutamatergic synapses (Taylor et al., 2014). These findings raised the intriguing possibility that increasing O-GlcNAc modification could lead to a dampening circuit activity in hyperexcitable conditions, such as epilepsy, and suggest that increasing O-GlcNAcylation could represent a novel therapeutic target.

Here, we demonstrate that an acute increase in protein O-GlcNAcylation significantly decreases ongoing PTX-induced epileptiform activity in area CA1 and limits subsequent PTXinduced hyperexcitability in both rat and mouse hippocampal slices. Using the HBP substrate GlcN alone, significant attenua- 
A

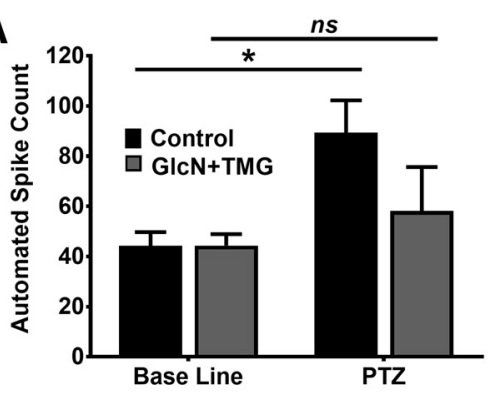

$\mathrm{D}_{1}$

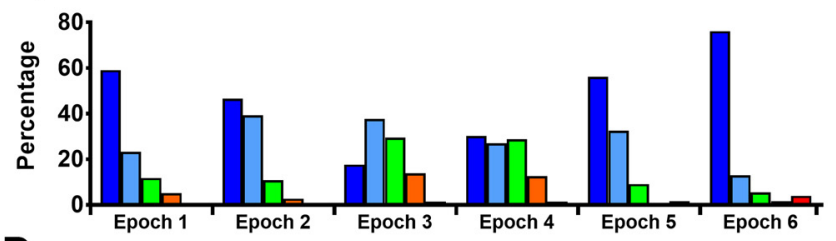

$D_{2}$
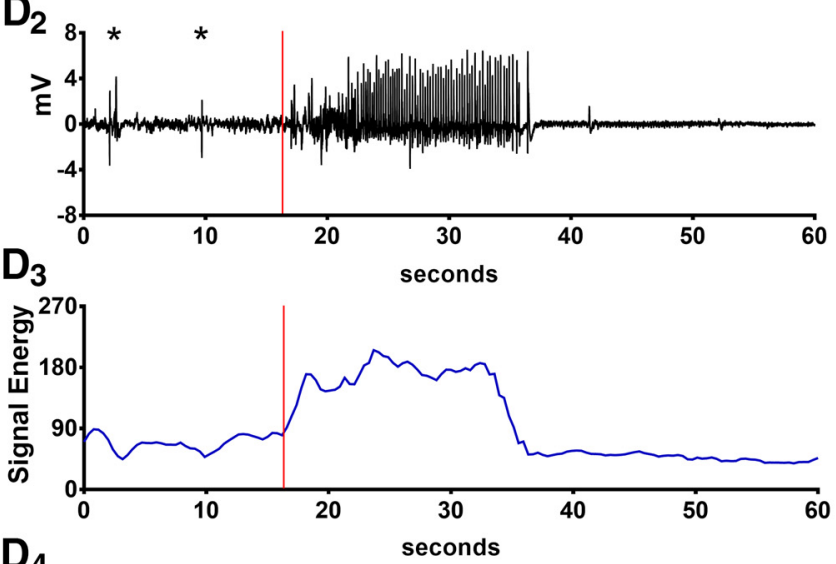

$\mathrm{D}_{4}$

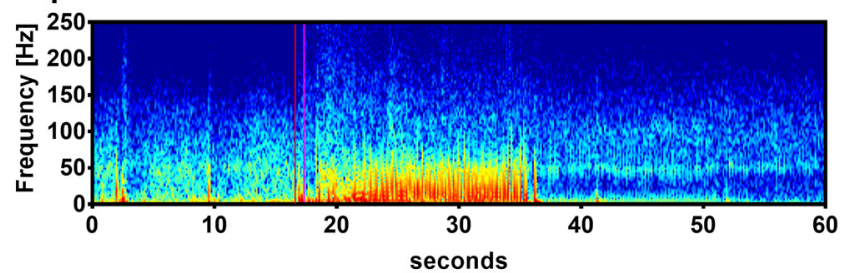

$F_{1}$

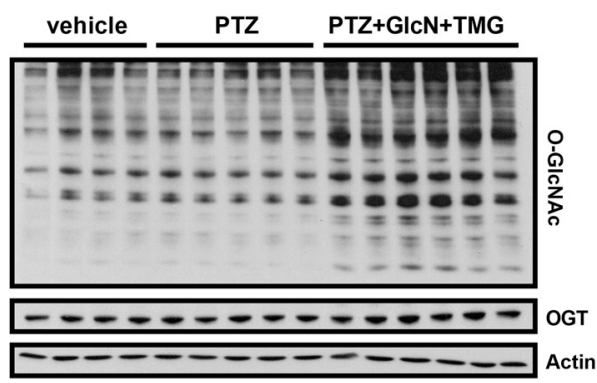

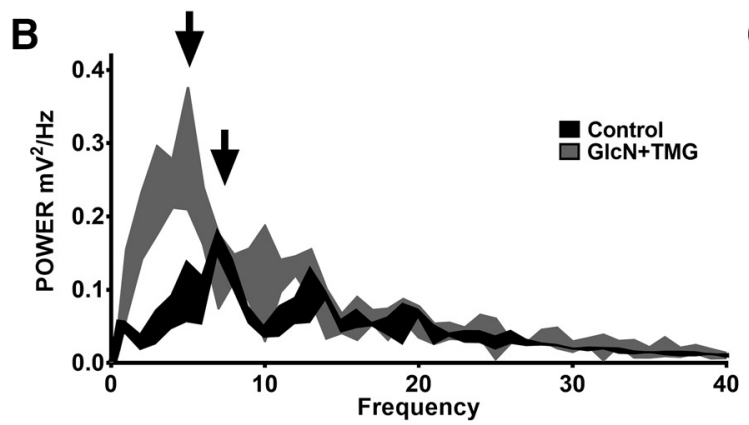

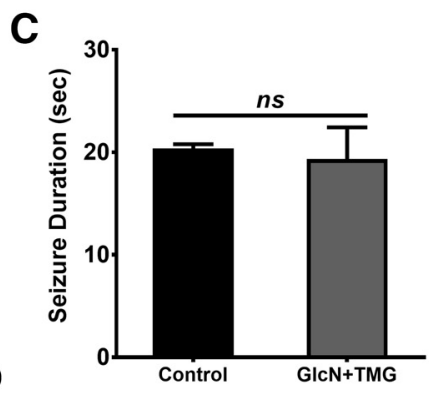

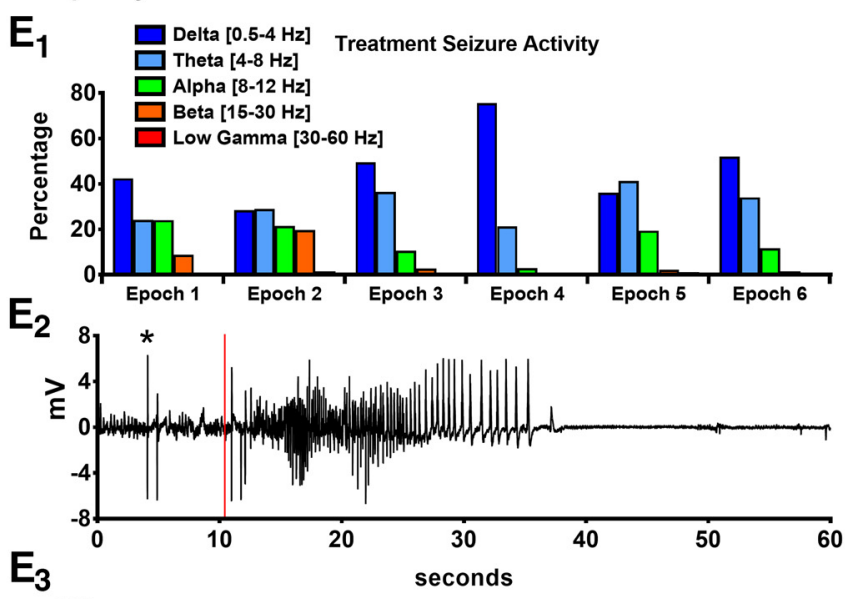

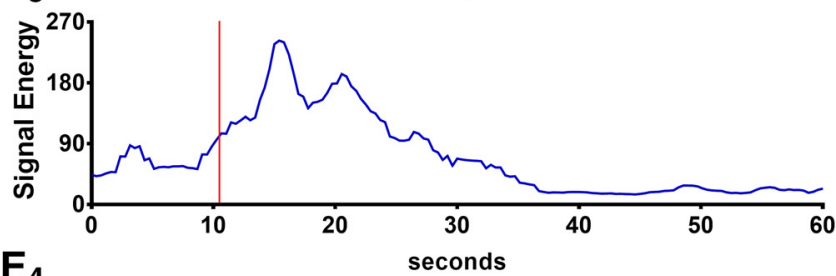

$\mathrm{E}_{4}$

$\mathbf{F}_{2}$

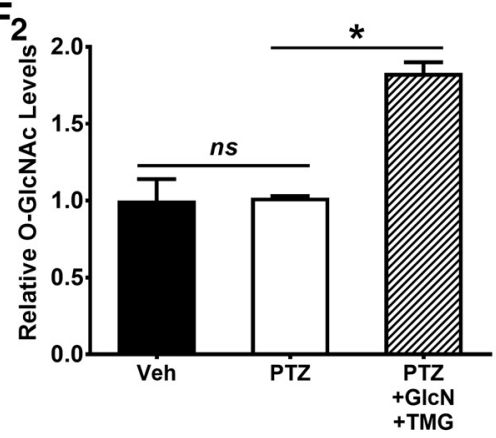

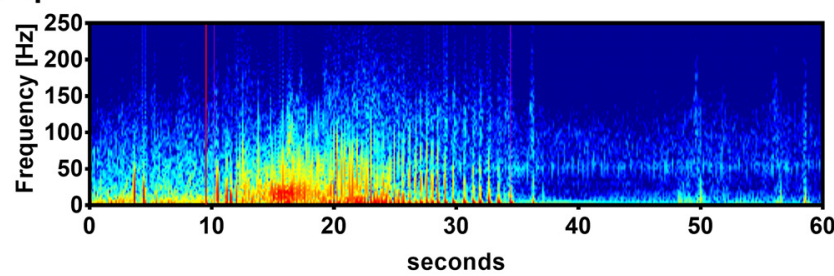

$F_{3}$

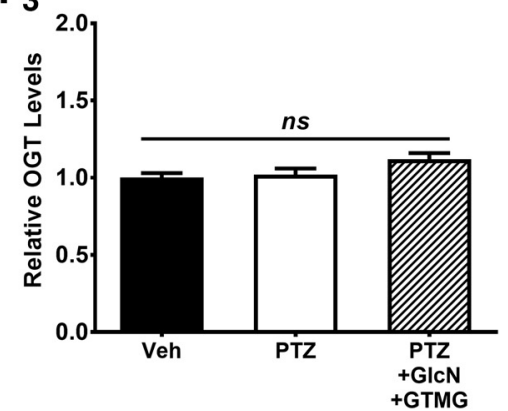

Figure 4. Increased 0-GICNAc levels protect against epileptiform activity in vivo. A, PTZ-induced interictal spikes is significantly increased in control animals $(n=15)$ but prevented in GlcN+TMG-pretreated animals $(n=14)$. $\boldsymbol{B}$, Spectral density analysis of seizures in saline-pretreated $(n=7$ of 15) versus GlcN+TMG-pretreated $(n=5$ of 14$)$ revealed altered frequency distributions across groups (peak power across groups denoted by top and bottom arrows). $C$, Comparison of cumulative seizure duration showed no significant differences across groups. $\boldsymbol{D}$, An example seizure in a saline-pretreated control mouse shows progressive redistribution of power across frequency domains during different stages of the seizure $\left(\boldsymbol{D}_{1}, \boldsymbol{D}_{2}\right)$, with the corresponding energy profile $\left(\boldsymbol{D}_{3}\right)$ and PSD plot $\left(\boldsymbol{D}_{4}\right)$ shown below. Red line indicates line-length calculated seizure onset. $\boldsymbol{E}$, An example seizure in a TMG + GlcN-pretreated mouse $\left(\boldsymbol{E}_{3}\right)$ shows contrasting frequency distributions during seizure progression, with power shifted into lower frequency bands following seizure onset $\left(\boldsymbol{E}_{1}\right.$, Epochs 3 and 4$)$. Once again, corresponding energy profile $\left(\boldsymbol{E}_{3}\right)$ and PSD plot $\left(\boldsymbol{E}_{4}\right)$ depict the line-length calculated seizure onset relative to EEG activity. $\boldsymbol{F}$, Western blot analysis of hippocampal tissue from EEG recorded mice was performed $\left(\boldsymbol{F}_{1}\right)$ showing significant increases in 0 -GlcNAc levels in GlcN+TMG-pretreated animals $\left(\boldsymbol{F}_{2} ;\right.$ PTX vs PTZ+TMG + GlcN, $\left.p<0.05\right)$. There was no significant difference in hippocampal $0 G$ levels across groups $\left(\boldsymbol{F}_{3} ;\right.$ PTZ vs $\mathrm{PTZ}+\mathrm{TMG}+\mathrm{GlCN}, p>0.05) .{ }^{*} p<0.05$. 
tion of ongoing hyperexcitability was observed. Indeed, a brief 10 min application of GlcN, which we have shown to transiently increase O-GlcNAc levels and induce O-GlcNAc LTD (Taylor et al., 2014), was capable of dampening ongoing PTX-induced hyperexcitability. Importantly, O-GlcNAc-mediated dampening of hyperexcitability requires GluA2-containing AMPARs, as a significant dampening effect is absent in slices from GluA2 KO mice. This suggests a conserved mechanism between O-GlcNAc LTD (Taylor et al., 2014) and O-GlcNAc-mediated dampening of hyperexcitability in area CA1. In recordings from CA3 pyramidal cells, both basal and PTX-induced spontaneous firing was attenuated by increasing O-GlcNAcylation. Similar to our observations in vitro, we found that increasing O-GlcNAcylation in vivo with an intraperitoneal injection of GlcN+TMG before administration of PTZ prevented the increase in interictal spikes observed in saline-pretreated mice. Furthermore, in a subset of GlcN+TMGpretreated mice that experienced fully convulsive seizures, spectral analysis showed peak power in lower frequency domains compared with seizures in saline-pretreated mice. Although the relationship between interictal spikes and convulsive seizures remains poorly understood (Staley and Dudek, 2006; Dudek and Staley, 2011; Staley et al., 2011), increasing O-GlcNAcylation appears to have a beneficial effect, at least at the level of interictal spiking activity. Although additional studies are needed to further test the therapeutic efficacy of GlcN+TMG in the treatment of both acute seizures as well as the formation of epileptic brain circuits, these preliminary results from both in vitro and in vivo model systems provide promising evidence for the benefits of O-GlcNAc-based treatment strategies.

Experimental epilepsy research has uncovered a complex relationship between synaptic plasticity and in vitro and in vivo models of seizures and epilepsy (Staley and Dudek, 2006; Kirschstein et al., 2007; Hellier et al., 2009), with the unifying hypothesis that intervening at the initial insult or onset of epileptogenesis is the most promising approach to treat the disease. Although our current work represents the first examination of therapeutic O-GlcNAcylation using in vitro and in vivo seizure models, others have shown therapeutic effects in multiple models of Alzheimer's disease (Borghgraef et al., 2013; Yuzwa et al., 2014). These findings reiterate the ability of pharmacologically increased O-GlcNAc to work to restore cellular function and homeostasis, whether to combat the effects of toxic tau aggregates or the formative hyperactivity leading to epileptic circuits in the brain. In addition, Wang et al. (2016) reported that conditional knock-out of OGT in mature forebrain excitatory neurons leads to the onset of an Alzheimer's neurodegenerative phenotype, including cell death, tau hyperphosphorylation, $\mathrm{A} \beta$ aggregation, and neuroinflammation. These mice exhibited memory deficits as well as tau and amyloid histopathologies, supporting the overarching hypothesis that endogenous O-GlcNAcylation plays a primary role in cell homeostasis and confers protection against neurodegenerative cell processes (Wang et al., 2016). A more recent study used genetic knockdown of OGA, resulting in constitutively elevated O-GlcNAc levels, and found deficits in contextual fear conditioning and attenuated LTP/ LTD in area CA1 (Yang et al., 2017). The conflicting results reported by Yang et al. (2017) and Taylor et al. (2014) could be attributed to the genetic versus pharmacological approach to increasing protein O-GlcNAcylation.

Given the rapidly growing list of O-GlcNAc-modified neuronal proteins, the mechanism(s) underlying O-GlcNAc-mediated circuit dampening is likely to be complex. Based upon our previously published report describing O-GlcNAc LTD (Taylor et al., 2014), the dampening effect on hyperexcitability is unlikely to involve decreased probability of glutamate release. Furthermore, because we induce epileptiform activity via blockade of $\mathrm{GABA}_{\mathrm{A}} \mathrm{Rs}$, an O-GlcNAc-mediated increase in GABAergic neuronal inhibition is not responsible, although our experiments do not rule out a potential role of $\mathrm{GABA}_{\mathrm{B}}$ Rs. However, O-GlcNAc dampening of hyperexcitability shares a conserved mechanism with O-GlcNAc LTD, since both require O-GlcNAcylation of the AMPAR GluA2 subunit. Furthermore, the possibility exists that our current findings stem from O-GlcNAc modulation of both synaptic and nonsynaptic proteins. To this end, Ruan et al. (2014) report O-GlcNAc modulation of the $\mathrm{K}^{+}$channel KCNQ3, which regulates excitability in a subset of hypothalamic neurons. Additionally, O-GlcNAcylation of CREB Ser40 shows sensitivity to $\mathrm{K}^{+}$induced increases in excitability of cultured neurons (Rexach et al., 2012). Although this contrasts with our current results where neither prolonged hyperexcitability in vitro (PTX) or increased interictal spikes in vivo (PTZ) affected global O-GlcNAc levels, we suggest that residue-specific biochemical analysis may reveal neuronal activity-dependent changes.

Within the expanding field of O-GlcNAc biology in brain, our current findings portray the HBP/O-GlcNAc metabolic system as a prime target to rapidly attenuate pathological hyperexcitability, as evidenced by both in vitro and in vivo neurophysiological experiments. With the advent of more advanced tools to measure and quantify protein O-GlcNAc both biochemically and physiologically, uncovering the molecular mechanisms of our current findings will provide further data in support of our hypothesis that increasing protein O-GlcNAcylation represents a potentially novel therapeutic target for the treatment of seizure disorders. Our results support the need for additional studies in more sophisticated preclinical epilepsy models to further advance the potential clinical use of GlcN and TMG as antiepileptic therapeutics.

\section{References}

Anggono V, Huganir RL (2012) Regulation of AMPA receptor trafficking and synaptic plasticity. Curr Opin Neurobiol 22:461-469. CrossRef Medline

Bond MR, Hanover JA (2015) A little sugar goes a long way: the cell biology of O-GlcNAc. J Cell Biol 208:869-880. CrossRef Medline

Borghgraef P, Menuet C, Theunis C, Louis JV, Devijver H, Maurin H, SmetNocca C, Lippens G, Hilaire G, Gijsen H, Moechars D, Van Leuven F (2013) Increasing brain protein O-GlcNAc-ylation mitigates breathing defects and mortality of Tau.P301L mice. PLoS One 8:e84442. CrossRef Medline

Cohen I, Miles R (2000) Contributions of intrinsic and synaptic activities to the generation of neuronal discharges in in vitro hippocampus. J Physiol 524:485-502. CrossRef Medline

Comer FI, Vosseller K, Wells L, Accavitti MA, Hart GW (2001) Characterization of a mouse monoclonal antibody specific for O-linked $\mathrm{N}$-acetylglucosamine. Anal Biochem 293:169-177. CrossRef Medline

Dudek FE, Staley KJ (2011) The time course of acquired epilepsy: implications for therapeutic intervention to suppress epileptogenesis. Neurosci Lett 497:240-246. CrossRef Medline

Esteller R, Echauz J, D’Alessandro M, Worrell G, Cranstoun S, Vachtsevanos G, Litt B (2005) Continuous energy variation during the seizure cycle: towards an on-line accumulated energy. Clin Neurophysiol 116:517-526. CrossRef Medline

Ge M, Wang D, Dong G, Guo B, Gao R, Sun W, Zhang J, Liu H (2013) Transient impact of spike on theta rhythm in temporal lobe epilepsy. Exp Neurol 250:136-142. CrossRef Medline

Hart GW (2014) Minireview series on the thirtieth anniversary of research on O-GlcNAcylation of nuclear and cytoplasmic proteins: nutrient regulation of cellular metabolism and physiology by O-GlcNAcylation. J Biol Chem 289:34422-34423. CrossRef Medline

Hellier JL, White A, Williams PA, Dudek FE, Staley KJ (2009) NMDA receptor-mediated long-term alterations in epileptiform activity in experimental chronic epilepsy. Neuropharmacology 56:414-421. CrossRef Medline 
Ivanov AI, Bernard C, Turner DA (2015) Metabolic responses differentiate between interictal, ictal and persistent epileptiform activity in intact, immature hippocampus in vitro. Neurobiol Dis 75:1-14. CrossRef Medline

Kanno T, Yaguchi T, Nagata T, Mukasa T, Nishizaki T (2010) Regulation of AMPA receptor trafficking by O-glycosylation. Neurochem Res 35:782788. CrossRef Medline

Kirschstein T, Bauer M, Muller L, Rüschenschmidt C, Reitze M, Becker AJ, Schoch S, Beck H (2007) Loss of metabotropic glutamate receptordependent long-term depression via downregulation of mGluR5 after status epilepticus. J Neurosci 27:7696-7704. CrossRef Medline

Korn SJ, Giacchino JL, Chamberlin NL, Dingledine R (1987) Epileptiform burst activity induced by potassium in the hippocampus and its regulation by GABA-mediated inhibition. J Neurophysiol 57:325-340. Medline

Lagerlöf O, Hart GW (2014) O-GlcNAcylation of neuronal proteins: roles in neuronal functions and in neurodegeneration. Adv Neurobiol 9:343366. CrossRef Medline

Liu K, Paterson AJ, Zhang F, McAndrew J, Fukuchi K, Wyss JM, Peng L, Hu Y, Kudlow JE (2004) Accumulation of protein O-GlcNAc modification inhibits proteasomes in the brain and coincides with neuronal apoptosis in brain areas with high O-GlcNAc metabolism. J Neurochem 89:1044-1055. CrossRef Medline

Löscher W (2009) Preclinical assessment of proconvulsant drug activity and its relevance for predicting adverse events in humans. Eur J Pharmacol 610:1-11. CrossRef Medline

Lüscher C, Malenka RC (2012) NMDA receptor-dependent long-term potentiation and long-term depression (LTP/LTD). Cold Spring Harb Perspect Biol 4:a005710. CrossRef Medline

Macauley MS, Whitworth GE, Debowski AW, Chin D, Vocadlo DJ (2005) O-GlcNAcase uses substrate-assisted catalysis: kinetic analysis and development of highly selective mechanism-inspired inhibitors. J Biol Chem 280:25313-25322. CrossRef Medline

Milikovsky DZ, Weissberg I, Kamintsky L, Lippmann K, Schefenbauer O, Frigerio F, Rizzi M, Sheintuch L, Zelig D, Ofer J, Vezzani A, Friedman A (2017) Electrocorticographic dynamics as a novel biomarker in five models of epileptogenesis. J Neurosci 37:4450-4461. CrossRef Medline

Ogawa M, Sawaguchi S, Kamemura K, Okajima T (2015) Intracellular and extracellular O-linked $\mathrm{N}$-acetylglucosamine in the nervous system. Exp Neurol 274:166-174. CrossRef Medline

Parker BL, Gupta P, Cordwell SJ, Larsen MR, Palmisano G (2011) Purification and identification of O-GlcNAc-modified peptides using phosphatebased alkyne CLICK chemistry in combination with titanium dioxide chromatography and mass spectrometry. J Proteome Res 10:1449-1458. CrossRef Medline

Rexach JE, Clark PM, Mason DE, Neve RL, Peters EC, Hsieh-Wilson LC (2012) Dynamic O-GlcNAc modification regulates CREB-mediated gene expression and memory formation. Nat Chem Biol 8:253-261. CrossRef Medline

Robel S, Buckingham SC, Boni JL, Campbell SL, Danbolt NC, Riedemann T,
Sutor B, Sontheimer H (2015) Reactive astrogliosis causes the development of spontaneous seizures. J Neurosci 35:3330-3345. CrossRef Medline

Ruan HB, Dietrich MO, Liu ZW, Zimmer MR, Li MD, Singh JP, Zhang K, Yin R, Wu J, Horvath TL, Yang X (2014) O-GlcNAc transferase enables AgRP neurons to suppress browning of white fat. Cell 159:306-317. CrossRef Medline

Schulz JB (2008) Update on the pathogenesis of Parkinson's disease. J Neurol 255 [Suppl 5]:3-7.

Staley KJ, Dudek FE (2006) Interictal spikes and epileptogenesis. Epilepsy Curr 6:199-202. CrossRef Medline

Staley KJ, White A, Dudek FE (2011) Interictal spikes: harbingers or causes of epilepsy? Neurosci Lett 497:247-250. CrossRef Medline

Tallent MK, Varghis N, Skorobogatko Y, Hernandez-Cuebas L, Whelan K, Vocadlo DJ, Vosseller K (2009) In vivo modulation of O-GlcNAc levels regulates hippocampal synaptic plasticity through interplay with phosphorylation. J Biol Chem 284:174-181. CrossRef Medline

Taylor EW, Wang K, Nelson AR, Bredemann TM, Fraser KB, Clinton SM, Puckett R, Marchase RB, Chatham JC, McMahon LL (2014) O-GlcNAcylation of AMPA receptor GluA2 is associated with a novel form of long-term depression at hippocampal synapses. J Neurosci 34:10-21. CrossRef Medline

Trinidad JC, Barkan DT, Gulledge BF, Thalhammer A, Sali A, Schoepfer R, Burlingame AL (2012) Global identification and characterization of both O-GlcNAcylation and phosphorylation at the murine synapse. Mol Cell Proteomics 11:215-229. CrossRef Medline

Wang AC, Jensen EH, Rexach JE, Vinters HV, Hsieh-Wilson LC (2016) Loss of O-GlcNAc glycosylation in forebrain excitatory neurons induces neurodegeneration. Proc Natl Acad Sci U S A 113:15120-15125. CrossRef Medline

Yang YR, Song S, Hwang H, Jung JH, Kim SJ, Yoon S, Hur JH, Park JI, Lee C, Nam D, Seo YK, Kim JH, Rhim H, Suh PG (2017) Memory and synaptic plasticity are impaired by dysregulated hippocampal O-GlcNAcylation. Sci Rep 7:44921. CrossRef Medline

Yuzwa SA, Vocadlo DJ (2014) O-GlcNAc and neurodegeneration: biochemical mechanisms and potential roles in Alzheimer's disease and beyond. Chem Soc Rev 43:6839-6858. CrossRef Medline

Yuzwa SA, Macauley MS, Heinonen JE, Shan X, Dennis RJ, He Y, Whitworth GE, Stubbs KA, McEachern EJ, Davies GJ, Vocadlo DJ (2008) A potent mechanism-inspired O-GlcNAcase inhibitor that blocks phosphorylation of tau in vivo. Nat Chem Biol 4:483-490. CrossRef Medline

Yuzwa SA, Shan X, Jones BA, Zhao G, Woodward ML, Li X, Zhu Y, McEachern EJ, Silverman MA, Watson NV, Gong CX, Vocadlo DJ (2014) Pharmacological inhibition of O-GlcNAcase (OGA) prevents cognitive decline and amyloid plaque formation in bigenic tau/APP mutant mice. Mol Neurodegener 9:42. CrossRef Medline

Zou L, Zhu-Mauldin X, Marchase RB, Paterson AJ, Liu J, Yang Q, Chatham JC (2012) Glucose deprivation-induced increase in protein O-GlcNAcylation in cardiomyocytes is calcium-dependent. J Biol Chem 287:34419-34431. CrossRef Medline 